\title{
How speakers select synthetic and analytic forms of English comparatives: An experimental study
}

Naomi Enzinna*

\begin{abstract}
This research examines the influence of prosodic shape, token frequency, and recency on comparative form preferences in English. To examine this, participants completed an unprimed and a primed forced-choice acceptabilityjudgment task. While the unprimed study's results show that comparative form selection is largely influenced by an adjective's prosodic shape and token frequency, the primed study shows that recency also plays a role in comparative form selection. More specifically, when primed with a synthetic comparative, participants were less likely to choose the comparative form that the adjective typically occurs in. These results are paralleled by the reaction time results, suggesting that recency of a synthetic comparative may cause either a facilitatory or inhibitory effect when selecting a comparative form.
\end{abstract}

Keywords. comparatives; prosodic shape; frequency; recency; priming

1. Introduction. In English, there are two comparative forms for adjectives: the synthetic comparative, formed with -er (e.g., easier, smarter), and the analytic comparative, formed with more (e.g., more famous, more clever). For many adjectives, English speakers prefer one form over the other. For example, easier is typically preferred over more easy, and more famous is typically preferred over famouser. These preferred comparative forms occur more frequently than the less preferred forms: In the Corpus of Contemporary American English (Davies 2008-), easier occurs over 30,000 times, while more easy occurs fewer than 100 times; and more famous occurs over 500 times, while famouser occurs only 3 times. Importantly, however, both forms occur. In this paper, I investigate what causes English speakers to select either the synthetic or the analytic comparative form, specifically examining the role of prosodic shape, token frequency, and most importantly, recency - the recent occurrence of a form in the discourse.

It is well known that an adjective's prosodic shape influences comparative form selection (Jespersen 1949, Cygan 1975, Bauer 1994, Leech \& Culpeper 1997, Lindquist 2000, among others). The number of syllables in an adjective is often a deciding factor. Monosyllabic adjectives are frequently preferred in the synthetic comparative form (e.g., smarter, kinder). Adjectives of three syllables or more almost always occur in the analytic comparative form (e.g., more intelligent, more important). However, for disyllabic adjectives, it is more complicated. Instead of the number of syllables, the word-final segments and the location of stress play a role. For instance, disyllabic adjectives ending in $y$ are usually preferred in the synthetic comparative form (e.g., easier), while disyllabic adjectives ending in a sibilant are usually preferred in the analytic form (e.g., more famous). However, previous studies have noted exceptions to these prosodic shape generalizations (Aranoff 1976, Ballinger 1991, Graziano-King 1999). For example, despite apt

\footnotetext{
* I would like to thank Draga Zec, Sam Tilsen, Miloje Despić, and Mats Rooth for their guidance and feedback; Sam Tilsen and Guandao Yang for creating the experiment website used in this research; and Bruce McKee, Stacy Dickerman, the Cornell Linguistics Department, the Cornell Phonetics Data Analysis Working Group, the Cornell Phonetics Lab, and the audience of the 2017 Annual Meeting of the Linguistic Society of America for their feedback and/or assistance. Author: Naomi Enzinna, Cornell University (nre23@cornell.edu).
} 
and chic being monosyllabic adjectives, more apt and more chic are preferred over apter and chicer.

According to Graziano-King (1999), these exceptions are due to apt and chic's low token frequency. She argues that high-frequency adjectives are more likely to be preferred in the synthetic comparative form, while low-frequency adjectives are more likely to be preferred in the analytic comparative form. For example, smart occurs in the COCA 25,799 times and chic occurs 3,044 times; for this reason, smarter is often preferred over more smart, and more chic is often preferred over chicer, despite both adjectives being monosyllabic. However, while chic's low token frequency explains why more chic is preferred over chicer, it does not explain when chicer still occurs.

One potential explanation is that recent forms can influence comparative form selection. If a speaker has recently heard chic or chicer, for instance, they may be more likely to say chicer. Some evidence of this can be found in the COCA: Chicer occurs 13 times (compared to 23 instances of more chic); in 10 out of those 13 instances, chicer occurred in a magazine-typically a fashion magazine - where being chic is likely to be discussed. This may suggest that recent forms in a speaker's environment can influence their comparative preferences. Similarly, recency of a comparative form may influence speakers to use that form. For example, if a speaker has recently heard a synthetic form (e.g., roomier), they may be more likely to use a synthetic form (e.g., chicer instead of more chic). Evidence from priming studies supports this possibility: In a study by Bock (1986), participants primed with various syntactic forms were more likely to produce the forms they were primed with. The same may be true for comparative form selection.

Thus, in this study I examine the influence of prosodic shape, frequency, and recency on speaker preferences for English comparatives. I address this by conducting two experiments. In the Experiment 1, I examine the validity of various prosodic shape and frequency generalizations, using a forced-choice acceptability judgment task. This experiment establishes a baseline for Experiment 2, which examines recency effects. In this experiment, I examine the influence of three types of recency on comparative form preferences: base recency, same-synthetic recency, and different-synthetic recency-described in (1).

(1) a. Base recency: the recent occurrence of the base adjective in a target comparative pair - e.g., for smarter and more smart, base recency would be the recent occurrence of smart.

b. Same-synthetic recency: the recent occurrence of the synthetic comparative form in a target comparative pair-e.g., for smarter and more smart, same-synthetic recency would be the recent occurrence of smarter.

c. Different-synthetic recency: the recent occurrence of a synthetic comparative form that has a base adjective that is different than that of the target comparative paire.g., for smarter and more smart, different-synthetic recency could be the recent occurrence of messier.

In order to make a direct comparison, the task and stimuli from Experiment 1 are used in Experiment 2 , adding only a priming component. The results show that, while prosodic shape and frequency influence comparative form preferences, these preferences are flexible, and recencyspecifically, same-synthetic recency (1b) and different-synthetic recency (1c)—plays a role.

The remainder of this paper is organized as follows: Experiment 1 - the unprimed study - is presented in Section 2, and Experiment 2-the primed study - is presented in Section 3. In Section 4 and 5, I discuss the results, and in Section 6, I conclude. 
2. Experiment 1: Examining the influence of prosodic shape and frequency. In this study, I examine the influence of an adjective's prosodic shape and token frequency on comparative form preferences. More specifically, I aim to answer the following questions: What comparative form do speakers prefer for monosyllabic adjectives, and for disyllabic adjectives ending in $y, l y, l e$, $o w, e r, n t$, a sibilant, and final stress ${ }^{1}$ Do comparative form preferences differ depending on whether the adjective has a high or low token frequency?

2.1. Method. In this study, participants completed a forced-choice acceptability judgment task. Participants were shown both the synthetic and analytic comparative form of an adjective on a computer screen and were asked which form they would be more likely to say in a conversation with a friend. An example of a single trial is shown in Figure 1. The task was completed through a website that recorded both the participants' judgments and their reaction times.

Participants were recruited through Amazon Mechanical Turk (AMT). 50 AMT workers completed the unprimed study. All AMT workers lived within the U.S. and were self-reported native English speakers over the age of 18.

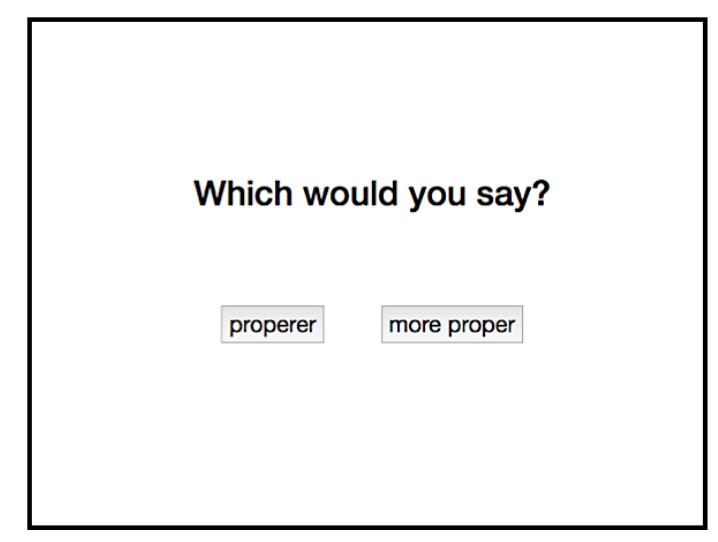

Figure 1. Example of a single trial in the unprimed study

The stimuli consisted 60 target adjectives, shown to participants in the synthetic and analytic comparative form (e.g., smarter and more smart). The target adjectives were controlled for prosodic shape and frequency. There were 12 monosyllabic adjectives and 48 disyllabic adjectives with 6 representing each of the following shapes: disyllabic adjectives ending in $y, l y, o w, l e, e r$, $n t$, a sibilant, and final stress. Half of the adjectives in each prosodic shape category were high frequency, and half were low frequency. The high-frequency adjectives occurred between approximately 10,000 to 20,000 times in the COCA and the 2000-2010 New York Times Annotated Corpus (NYT; Sandhaus 2008-). The low-frequency adjectives occurred between approximately 100 to 1,000 times in the COCA and NYT corpora.

To distract participants from the experiment's purpose, 120 adjective pairs were used as fillers. The adjectives in a pair shared a base adjective and had comparable meanings (e.g., uncool and not cool). Of the filler pairs, 40 included two acceptable adjectives (e.g., illegal and not legal), 40 included 1 acceptable adjective (e.g., blurry and *blurrous), and 40 included 0 acceptable adjectives (e.g., *disworn and *inworn). The pairs with 1 acceptable adjective were used in the study to ensure that participants were paying attention during the task. No participant

\footnotetext{
${ }^{1}$ These prosodic shapes were found in the previous literature to be relevant.
} 
was removed from the study for this reason, as no participant had more than two incorrect responses (38/40). There were 180 adjective pairs in total, randomized for each participant.

2.2. RESUlts. To examine whether prosodic shape influences comparative form preferences, a chi-squared test of independence was performed to test whether comparative form and prosodic shape are independent. There was a significant relationship between comparative form and prosodic shape, $\chi^{2}(8, N=2898)=855.28, p<0.001$. Monosyllabic adjectives, and disyllabic adjectives ending in $y$ and $l y$ were more likely to form the comparative synthetically $(r>7.69){ }^{2}$ Disyllabic adjectives ending in $e r, n t$, a sibilant, and final stress were less likely to form the comparative synthetically $(r<-6.94)$. Disyllabic adjectives ending in le were slightly less likely to form the comparative synthetically, and disyllabic adjectives ending in $o w$ were slightly more likely to form the comparative analytically $(-2.39<r<3.41)$. Therefore, results suggest that comparative form preferences are influenced by prosodic shape.

To examine the influence of frequency, a chi-squared test of independence was performed to test whether comparative form and frequency are independent. There was a significant relationship between comparative form and frequency, $\chi^{2}(1, N=2898)=72.21, p<0.001$. Therefore, comparative form and frequency are not independent; high-frequency adjectives were more likely to form the comparative synthetically $(r>4.97)$ than low-frequency adjectives $(r<-4.81)$. Despite this, high-frequency adjectives were more likely to form the analytic comparative than the synthetic comparative because certain prosodic shapes are not preferred in the synthetic form. Therefore, results suggest that comparative form preferences are influenced by frequency.

To examine whether frequency influences all prosodic shapes, a simple linear regression was conducted for each prosodic shape to test whether frequency significantly predicted the proportion of participants who chose the synthetic form (\%Synthetic). The results from the regression indicate that frequency only influenced comparative form preferences for monosyllabic adjectives, and disyllabic adjectives ending in $l y$. For monosyllabic adjectives, $78 \%$ of the variability in \%Synthetic can be accounted for with frequency $(F(2,9)=32.1, p<0.001)$. A monosyllabic adjective's predicted \%Synthetic is equal to $-62+15(\log$ frequency $) \%$; thus, an adjective's \%Synthetic increased $15 \%$ with each increase in log frequency. Similarly, for disyllabic adjectives ending in $l y, 97.6 \%$ of the variability in \%Synthetic can be accounted for with frequency $(F(2,4)=162, p<0.001)$. A disyllabic ly adjective's predicted \%Synthetic is equal to $-36.61+13(\log$ frequency $) \%$, meaning an adjective's \%Synthetic increased $13 \%$ with each increase in log frequency. For all other prosodic shapes, comparative form could not be predicted with frequency. Therefore, frequency influences comparative form preferences for some prosodic shapes, not all. These results are displayed in Figure 2.

In order to simplify and better understand the trends seen in Experiment 2's results, the prosodic shape categories were grouped into three different preference groups, shown in Table 1. These groups were created based on observational differences, as well as differences in standardized residuals, as discussed above.

\begin{tabular}{r|l} 
Preference Group & Description \\
\hline -er preference & monosyllabic adjectives; disyllabic adjectives ending in $y$ or $l y$ \\
\hline no preference & disyllabic adjectives ending in $o w$ or $l e$ \\
\hline more preference & disyllabic adjectives ending in $e r, n t$, a sibilant, or final stress
\end{tabular}

Table 1. Description of preference groups

\footnotetext{
${ }^{2} r$ is used here to represent the standardized residuals.
} 


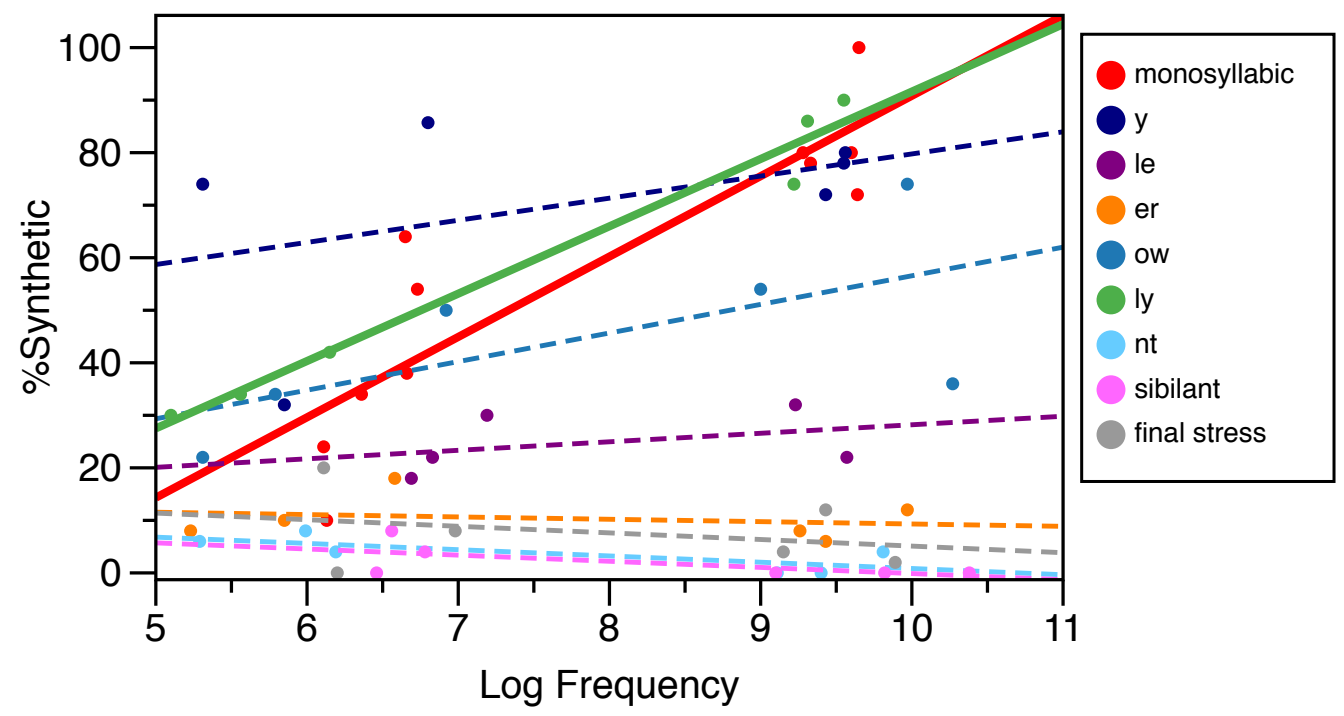

Figure 2. \%Synthetic by prosodic shape and frequency: Monosyllabic adjectives and disyllabic adjectives ending in $l y$ (red and green solid lines) were significantly influenced by frequency.

To compare preference groups, a chi-squared test of independence was performed to test whether comparative form and preference group are independent. There was a significant relationship between comparative form and preference group, $\left.\chi^{2}(2, N=2898)=810.03, p<0.001\right)$. Therefore, comparative form and preference group are not independent; -er-preference adjectives are more likely to form the comparative synthetically $(r>16.25)$, and more-preference adjectives are less likely to form the comparative synthetically $(r<-16.52)$. A significant relationship was not shown for no-preference adjectives $-r<-0.90$ ). This result is illustrated in Figure 3.

Results also show that frequency influences \%Synthetic for -er-preference adjectives only. For each preference group, a simple linear regression was calculated to test whether frequency significantly predicted \%Synthetic. For -er-preference adjectives, $60 \%$ of the variability in $\%$ Synthetic can be accounted for with frequency $(F(2,21)=32.4, p<0.001)$. An -er-preference adjective's predicted \%Synthetic is equal to $-24+11(\log$ frequency $) \%$; thus, an -er-preference adjective's \%Synthetic increased $11 \%$ with each increase in $\log$ frequency. For the no-preference and more-preference groups, comparative form could not be predicted with frequency.

2.3. SUMMARY. Experiment 1's results show that prosodic shape and frequency influence comparative form selection. Regarding prosodic shape, monosyllabic and disyllabic adjectives ending in $y$ and $l y$ were preferred in the synthetic form (-er preference adjectives). Disyllabic adjectives ending in $e r, n t$, a sibilant, and final stress were preferred in the analytic form (morepreference adjectives). Disyllabic adjectives ending in ow and le were not strongly preferred in either form (no-preference adjectives). Regarding frequency, for the following prosodic shapes, high-frequency adjectives were preferred in the synthetic form, and low frequency adjectives were preferred in the analytic form: monosyllabic adjectives, and disyllabic adjectives ending in $l y$. All other prosodic shapes were not influenced by frequency.

3. Experiment 2: Examining the influence of recency. In this study, I examine whether recent forms can influence comparative form preferences. Specifically, I address the following: Does base, same-synthetic, and/or different-synthetic recency - described in (1) - increase preference for the synthetic comparative form? 


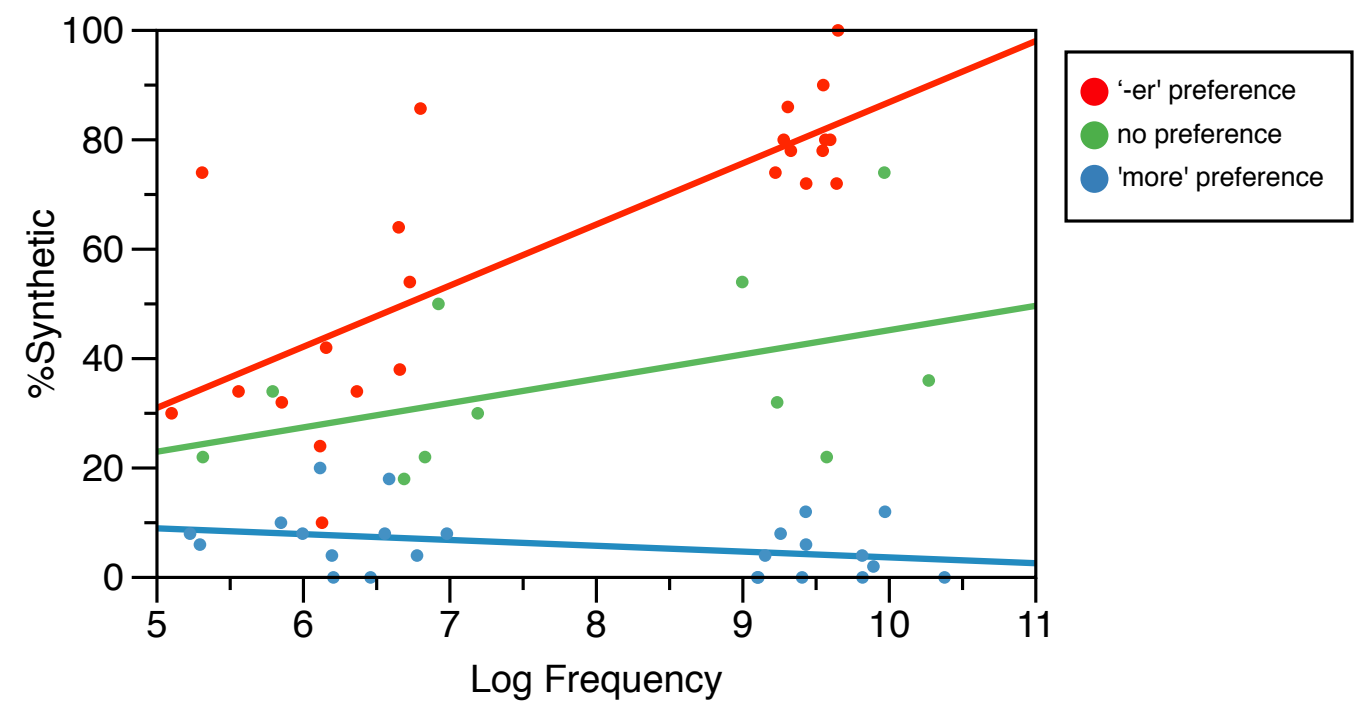

Figure 3. \%Synthetic by preference group and frequency: Frequency influences -er-preference adjectives, and does not influence no-preference and more-preference adjectives.

3.1. METHOD. In this study, participants completed the same forced-choice acceptability judgment task as in the unprimed study; however, before each task screen (Figure 4b), participants were shown a priming screen (Figure 4a), in which they read a single word on the screen before continuing to the task. An example of a single trial is shown in Figure 4. The target and filler adjective pairs were the same as in the unprimed study.

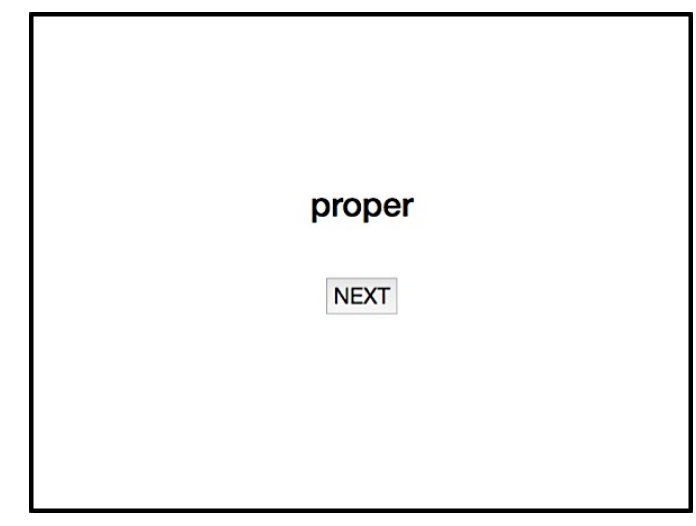

(a) Priming screen

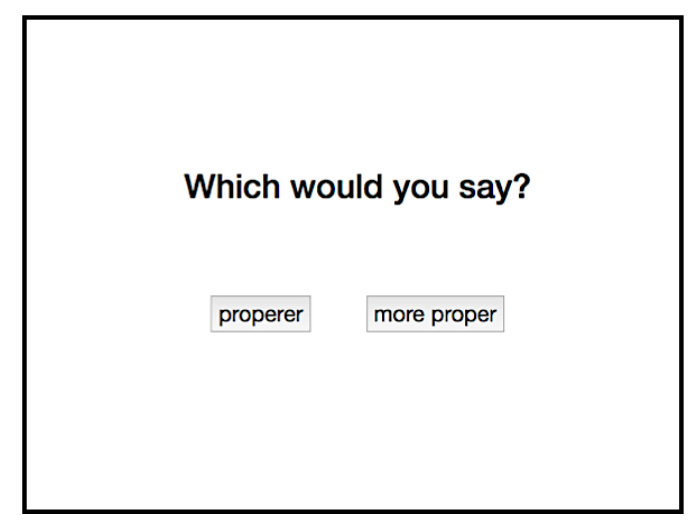

(b) Task screen

Figure 4. Example of a single trial during the primed study

There were three prime types for each target adjective: a base-only prime (to test base recency), a same-synthetic prime (to test same-synthetic recency), and a different-synthetic prime (to test different-synthetic recency). For example, the target pair famouser and more famous was primed with the base-only prime famous, the same-synthetic prime famouser, and the differentsynthetic prime roomier.

The different synthetic primes were chosen based on semantic and prosodic shape dissimilarity to the target adjective. To ensure prosodic shape dissimilarity, no target pair was primed with a synthetic comparative of the same prosodic shape category. To ensure semantic dissimilarity, semantic distances of primes to target adjectives were measured using global vectors for 
word representations (GloVe) and the Google News corpus (Pennington et al. 2014); each target was paired with a prime that had a semantic distance greater than the mean distance of all primes and targets (3.77 out of 10). Additionally, all different synthetic primes had a token frequency that fell between that of the high and low frequency adjectives in the synthetic form (between 100 and 200 instances approximately in the NYT and COCA corpora).

As with the target primes, fillers were primed with base, same, and different primes. The base prime was the base adjective that both adjectives in a pair share. The same prime was one of the two adjectives in the pair. The different prime shared an affix with one of the adjectives in the pair. For example, for the filler pair unkind and not kind, the three primes were kind (base), unkind (same), and unpaid (different). Thus, there were three prime types for all adjective pairs.

Several measures were taken to randomize the primed study. Similar to the unprimed study, all pairs appeared in a randomized order. Further, to ensure that participants only judged an adjective pair once, there were three versions of the primed study, and each of the three versions had $1 / 3$ of each prime type. Last, half of the same and different filler primes primed toward an acceptable adjective, and half toward an unacceptable adjective.

50 AMT workers completed each of the three versions of the study (150 total). No participant who completed the primed study also completed the unprimed study or another version of the primed study. All AMT workers lived within the U.S. and were self-reported native English speakers over the age of 18 .

3.2. RESUlTS: SAME-SYNTHETIC RECENCY. A chi-squared goodness-of-fit test was performed to test whether the distribution of comparative forms per preference group and frequency was equal in the unprimed and same-synthetic primed studies. The distribution of comparative forms differs depending on whether participants were primed with a same-synthetic prime or not primed, $\chi^{2}(11, N=2898)=60.70, p<.001$. More specifically, when primed a same-synthetic prime, high-frequency -er-preference and no-preference adjectives were preferred in the synthetic form fewer times ( $r=-2.42$ and $r=-2.87$, respectively) and the analytic more times $(r=4.88$ and $r=$ 2.53 , respectively) than when unprimed. Contrastingly, high-frequency more-preference adjectives were preferred in the synthetic form more than when unprimed $(r=3.47)$. Priming did not influence low-frequency adjectives.

Therefore, priming participants with a same-synthetic prime influenced comparative form preferences: high-frequency adjectives that are preferred in the synthetic form (-er-preference and no-preference adjectives) became less likely to form the comparative synthetically, and highfrequency adjectives that are not preferred in the synthetic form (more-preference adjectives) became more likely to be preferred in the synthetic form. Figure 5 illustrates, for each preference group and frequency, the \%Synthetic difference between the same-synthetic primed and unprimed studies.

3.3. Results: DifFERENT-SYNTHETIC RECENCY. A chi-squared goodness-of-fit test was performed to test whether the distribution of comparative forms per preference group and frequency was equal in the unprimed and different-synthetic primed studies. The distribution of comparative forms differs depending on whether participants were primed with a different-synthetic prime or not primed, $\chi^{2}(11, N=2899)=28.68, p=.002$. More specifically, when primed a different-synthetic prime, high-frequency -er-preference adjectives were preferred in the analytic form more $(r=4.00)$ than when unprimed. Contrastingly, high-frequency more-preference adjectives were preferred in the synthetic form more than when unprimed $(r=2.65)$. Low-frequency adjectives and no-preference adjectives were not influenced by priming. 


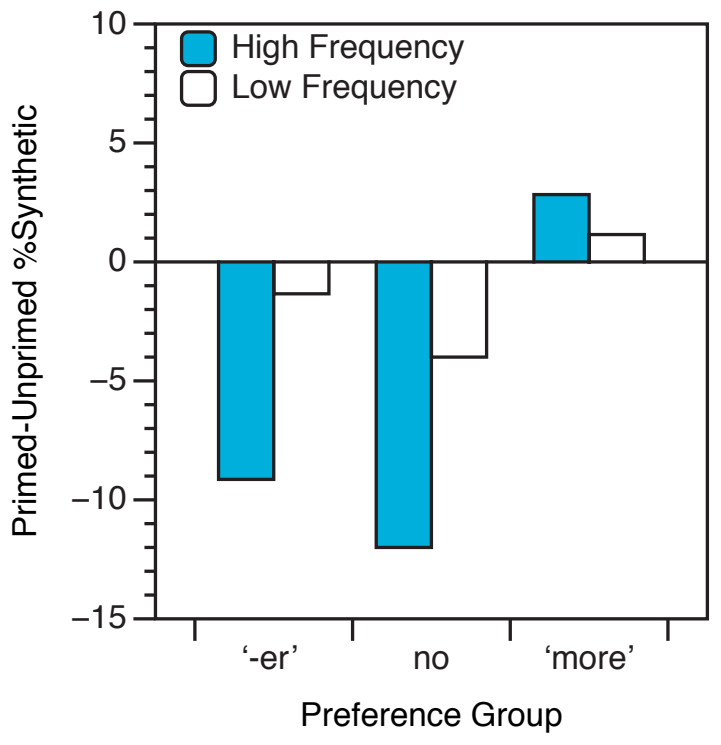

Figure 5. Same-synthetic recency: \%Synthetic significantly decreased for high-frequency -erpreference and no-preference adjectives and significantly increased for high-frequency morepreference adjectives when primed with a same-synthetic prime.

Therefore, priming participants with a different-synthetic prime influenced comparative form preferences: high-frequency -er-preference adjectives were less likely to be preferred in the synthetic comparative form, and high-frequency more-preference adjectives were more likely to preferred in the synthetic comparative form. Figure 6 illustrates, for each preference group and frequency, the \%Synthetic difference between the different-synthetic primed and unprimed studies.

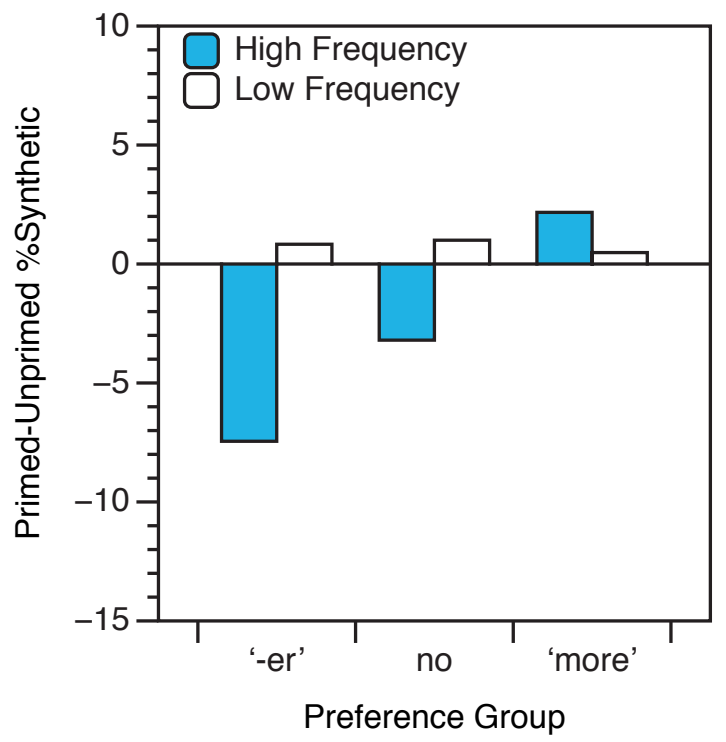

Figure 6. Different-synthetic recency: \%Synthetic significantly decreased for high-frequency -erpreference adjectives and significantly increased for high-frequency more-preference adjectives when primed with a different-synthetic prime. 
3.4. RESULTS: BASE RECENCY. A chi-squared goodness-of-fit test was performed to test whether the distribution of comparative forms per preference group and frequency was equal in the unprimed and base-only primed studies. There is no evidence of the comparative forms being distributed differently in the two studies, $\chi^{2}(11, N=2897)=16.86, p=.11$. Therefore, priming participants with a base-only prime did not influence comparative form preferences. Figure 7 shows, for each preference group and frequency, the \%Synthetic difference between the baseonly primed and unprimed studies. ${ }^{3}$

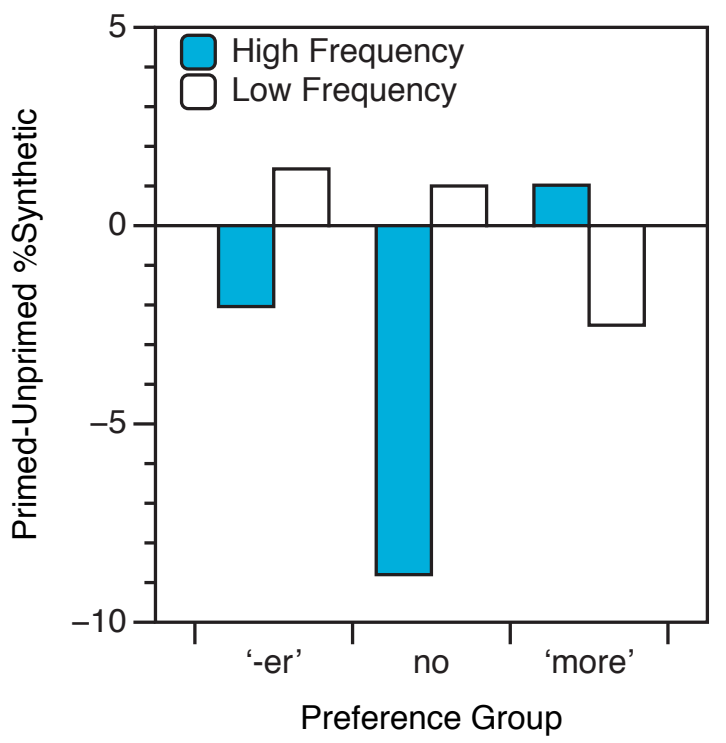

Figure 7. Base recency: Priming did not significantly influence comparative form preferences.

3.5. SUMmaRY. Experiment 2's results show that same-synthetic recency and different-synthetic recency influence comparative form preferences. Priming with a same-synthetic or differentsynthetic prime increased preference for the synthetic form for high-frequency more-preference adjectives (which typically do not occur in the synthetic form) and, unexpectedly, decreased preference for the synthetic form for high-frequency -er-preference and no-preference adjectives (which typically do occur in the synthetic form). Priming with a base-only prime did not influence comparative form preferences. Of the three recency types tested, same-synthetic recency had the greatest impact on comparative form preferences (as illustrated in Figure 8).

These results leave us with two questions. First, why does priming influence -er-preference and more-preference adjectives differently? Second, why does recency only influence highfrequency adjectives? I address the first question in Section 4 and the second question briefly in Section $5 .{ }^{4}$

\footnotetext{
${ }^{3}$ It should be noted that, although it is not statistically significant, high-frequency disyllabic adjectives ending in $l e$ showed a large decrease in preference for the synthetic form when primed with a base-only prime. Correspondingly, no-preference adjectives had a large decrease in preference for the synthetic form when primed with a base-only prime. This result seems to align with the insignificant-but-large reaction time results for this preference group in Section 4.4. This suggests that priming may be influencing this group in some way.

${ }^{4}$ For additional information and figures from both Experiment 1 and 2, refer to the LSA presentation slides (https://www.researchgate.net/publication/317286127_How_speakers_select_synthetic_and_analytic_forms_of_Eng lish_comparatives_an_experimental_study).
} 


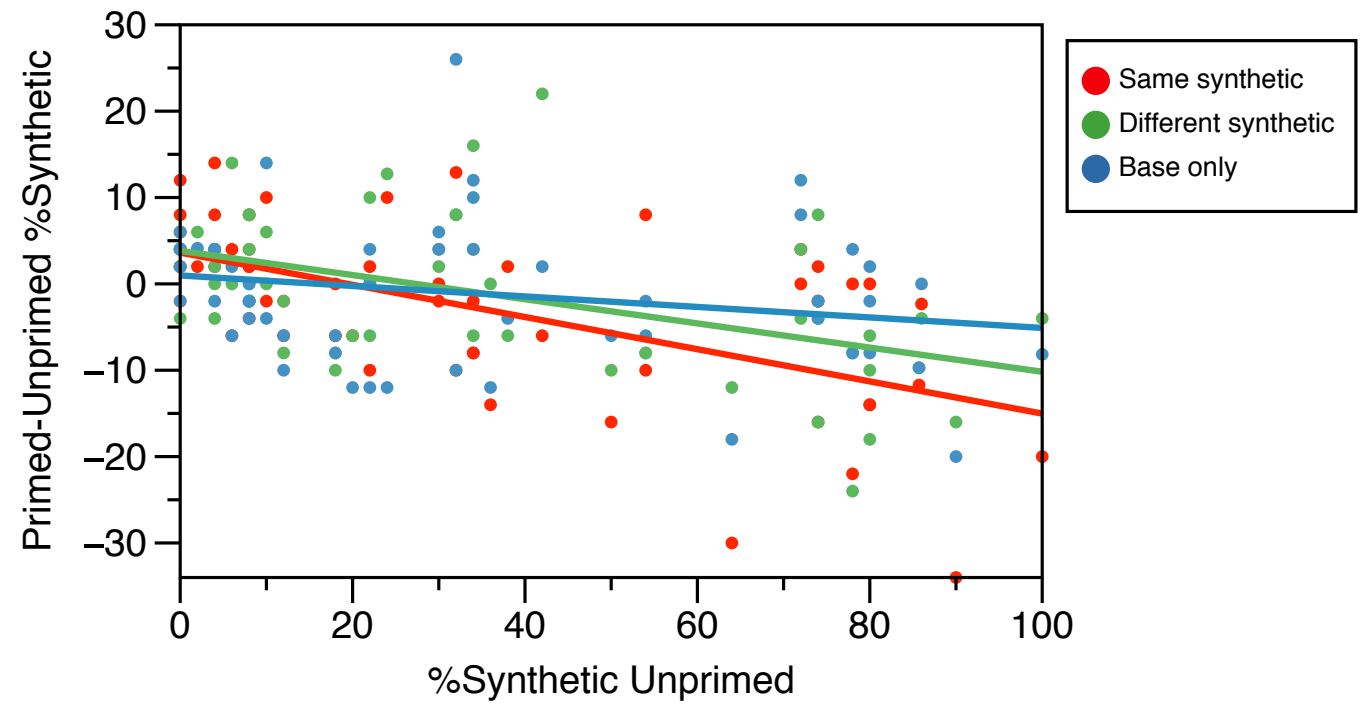

Figure 8. Difference in \%Synthetic by prime type: Same-synthetic and different-synthetic primes influenced comparative form preferences, while the base-only prime did not.

\section{Why does priming affect -er-preference and more-preference adjectives differently?}

Priming can cause two types of effects: a facilitatory effect and an inhibitory effect. When priming causes facilitation, participants' reactions are more accurate and/or faster. When priming causes inhibition (also known as "negative priming"), participants' reactions are more errorprone and/or slower (Mayr and Buchner 2007).

Regarding the primed study's (Experiment 2) acceptability-judgment task results, priming participants with a same-synthetic or different-synthetic prime facilitated, or increased, selection of the synthetic comparative form for the more-preference adjectives. For example, when participants were primed with famouser and then asked whether they would be more likely to say famouser or more famous, more participants chose famouser over more famous than when unprimed. This result was expected because participants were primed with a synthetic comparative.

Contrastingly, for -er-preference and, sometimes, no-preference adjectives, priming with a synthetic prime inhibited, or decreased, selection of the synthetic form. For example, when participants were primed with cheaper and then asked whether they would be more likely to say cheaper or more cheap, more participants chose more cheap over cheaper than when unprimed. This result was unexpected because participants were being primed with a synthetic form and because -er-preference adjectives and no-preference adjectives often occur in the synthetic form. Therefore, this result may be interpreted as a type of inhibition "error" effect.

Examining participants' reaction times (RTs) ${ }^{5}$ further supports this interpretation of the results. When priming participants with a same-synthetic prime, there was no difference in reaction times between the primed and unprimed studies for more-preference adjectives. However, for -er-preference and no-preference adjectives, participants' had longer RTs when they selected the

\footnotetext{
${ }^{5}$ RT outliers were removed in two ways: First, to remove extreme outliers, RTs below the 2.5 th and above the 97.5th quantiles were excluded, leaving 95\% of the data to analyze (minimum RT: 770 ms, maximum RT: 5077 $\mathrm{ms}$ ). Second, for each participant, I calculated z-scores for their RTs and removed all z-scores greater than 3 or less than -3 . The remaining z-scores were used in the analysis; z-scores were used instead of RTs to normalize RTs and account for differences between participants.
} 
synthetic comparative form, compared to the unprimed study. In other words, priming with a same-synthetic prime inhibited selection of the synthetic comparative form for -er-preference and no-preference adjectives - causing participants to either (1) slow down to select the synthetic comparative form (causing longer RTs), or (2) select the analytic comparative form (causing an increased preference for the analytic form). For more-preference adjectives, RTs were shorter than RTs from the unprimed study, regardless of comparative preference, and there was an increased preference for the synthetic form; therefore, facilitation, not inhibition, occurred.

Priming with a different-synthetic prime caused additional inhibition effects, however. RTs were longer when participants were primed than unprimed, regardless of an adjective's preference group or comparative form selection. This result is expected because previous studies show that inhibition occurs when there are semantic differences between primes and target words (Raveh 2002). However, the facilitation/inhibition analysis used to explain the results of the same-synthetic primed study is still applicable because the acceptability-judgment task results are similar for both studies. Also, when comparing the RTs of the three preference groups (-er, no, and more preference) within the different-synthetic primed study, RTs were longer for -erpreference adjectives, compared to the RTs of the more-preference adjectives. This result suggests that priming with a different-synthetic prime affects -er-preference and no-preference adjectives differently than more-preference adjectives.

Below, I analyze RTs by preference group, in order to compare each preference groups' RTs to the RTs of the unprimed study. Then, I summarize the RT results.

4.1. ANALYSIS OF REACTION TIMES FOR -ER-PREFERENCE ADJECTIVES. A two-way ANOVA was conducted to examine the effect of prime type and comparative preference on RTs (in z-scores) ${ }^{6}$ for -er-preference adjectives. There was a statistically significant interaction between the effects of prime type and comparative preference on $\mathrm{RT}, F(3,4429)=2.09, p=.05$.

Post hoc comparisons using the Tukey HSD test indicated that the mean RT for -erpreference adjectives primed with a same-synthetic prime was significantly higher when the participant chose a synthetic form $(\mathrm{M}=-.080, \mathrm{~S}=.893)$ than when they chose an analytic form (M $=-.209, \mathrm{~S}=.865)$. Also, the mean RT for -er-preference adjectives was significantly lower when the participant chose an analytic form and was primed with a same-synthetic prime than when a participant was not primed and chose an analytic form $(\mathrm{M}=.026 \mathrm{~ms}, \mathrm{~S}=.902 \mathrm{~ms})$ or a synthetic form $(\mathrm{M}=-.036, \mathrm{~S}=.913)$. In other words, participants who were primed with a same-synthetic prime took longer to choose a synthetic form compared to an analytic form, and participants primed with a same-synthetic prime took less time to choose an analytic form than an unprimed participant choosing either form.

Post hoc comparisons also indicated that participants primed with a different-synthetic prime had longer RTs when choosing a synthetic form $(\mathrm{M}=.228, \mathrm{~S}=.935)$ or an analytic form $(\mathrm{M}=$ $.307, \mathrm{~S}=.941)$ than when unprimed and choosing a synthetic form $(\mathrm{M}=-.036, \mathrm{~S}=.913)$ or analytic form $(\mathrm{M}=.026 \mathrm{~ms}, \mathrm{~S}=.902)$. In other words, participants took longer to react if they were primed with a different-synthetic prime, regardless of whether they chose a synthetic or analytic form.

Last, post hoc comparisons indicated that there were no significant differences between analytic and synthetic form $\mathrm{RTs}$ for both the unprimed $(\mathrm{M}=.026 \mathrm{~ms}, \mathrm{~S}=.902 ; \mathrm{M}=-.036, \mathrm{~S}=.913$; respectively) and base-only prime studies $(\mathrm{M}=-.034, \mathrm{~S}=.915 ; \mathrm{M}=-.073, \mathrm{~S}=.911$; respective-

\footnotetext{
${ }^{6}$ The analyses were conducted using both mean RTs and RT z-scores. I report the statistics on the RT z-scores because this accounts for differences in RTs between participants. However, all significant results that are reported here were significant regardless of whether the analysis was conducted on mean RTs or RT z-scores.
} 
ly). There were also no significant differences between the unprimed study RTs and the baseonly primed study RTs. In other words, when participants were not primed, there were no significant differences between choosing a synthetic versus an analytic form. Participants who were primed with a base-only prime reacted similarly to participants who were unprimed.

A simple main effects analysis showed a main effect for prime type, $F(3,4429)=42.01, p<$ .001 ; participants primed with a same-synthetic prime had shorter RTs than unprimed participants, while participants primed with a different-synthetic prime had longer RTs than all other prime types. There was also a main effect for comparative preference, $F(1,4429)=3.64, p=.05$; RTs were longer when participants chose a synthetic form than an analytic form.

4.2. ANALYSIS OF REACTION TIMES FOR NO-PREFERENCE ADJECTIVES. A two-way ANOVA was conducted to examine the effect of prime type and comparative preference on RTs for nopreference adjectives. There was a statistically significant interaction between the effects of prime type and comparative preference on $\mathrm{RT}, F(3,2212)=6.04, p<.001$.

Post hoc comparisons using the Tukey HSD test indicated that the mean RT for nopreference adjectives primed with a same-synthetic prime was significantly higher when the participant chose a synthetic form $(\mathrm{M}=.176, \mathrm{~S}=1.075)$ than when they chose an analytic form (M $=-253, \mathrm{~S}=.864)$. Also, the mean RT for no-preference adjectives was significantly lower when the participant chose an analytic form and was primed with a same-synthetic prime than when a participant was not primed and chose an analytic form $(\mathrm{M}=-.009, \mathrm{~S}=.879)$. In other words, participants who were primed with a same-synthetic prime took longer to choose a synthetic form compared to an analytic form, and participants primed with a same-synthetic prime took less time to choose an analytic form than a participant who was unprimed.

Post hoc comparisons also indicated that participants primed with a different-synthetic prime had longer RTs when choosing a synthetic form $(\mathrm{M}=.290, \mathrm{~S}=.951)$ or an analytic form $(\mathrm{M}=$ $.379, \mathrm{~S}=.908)$ than when unprimed and choosing a synthetic form $(\mathrm{M}=-.055, \mathrm{~S}=.903)$ or analytic form $(\mathrm{M}=-.009, \mathrm{~S}=.879)$. In other words, participants took longer to react if they were primed with a different-synthetic prime, regardless of whether they chose a synthetic or analytic form.

Last, post hoc comparisons indicated that there were no significant differences between analytic and synthetic form RTs for both the unprimed $(\mathrm{M}=-.009, \mathrm{~S}=.879 \mathrm{~ms} ; \mathrm{M}=-.055 \mathrm{~ms}, \mathrm{~S}=$ $.903 \mathrm{~ms}$; respectively) and base-only primed studies $(\mathrm{M}=-.171, \mathrm{~S}=.838 ; \mathrm{M}=.036, \mathrm{~S}=.924$; respectively). There were also no significant differences between the unprimed study RTs and the base-only prime study RTs. In other words, when participants were not primed, there were no significant differences between choosing a synthetic versus an analytic form. Participants who were primed with a base-only prime reacted similarly to participants who were unprimed.

Simple main effects analysis showed a main effect for prime type, $F(3,2212)=21.31, p<$ .001 ; participants primed with a different-synthetic prime had longer RTs than all other prime types. There was also a main effect for comparative preference, $F(1,2212)=9.83, p=.002$; RTs were longer when participants chose a synthetic form than an analytic form.

4.3. ANALYSIS OF REACTION TIMES FOR MORE-PREFERENCE ADJECTIVES. A two-way ANOVA was conducted to examine the effect of prime type and comparative preference on RT for morepreference adjectives. There was not a statistically significant interaction between the effects of prime type and comparative preference on $\mathrm{RT}, F(3,4529)=0.13, p=.93$.

Simple main effects analysis showed a main effect for prime type, $F(3,4529)=19.2, p<$ .001 ; participants primed with a same-synthetic prime had shorter RTs than unprimed participants, while participants primed with a different-synthetic prime had longer RTs than all other 
prime types. There was also a main effect for comparative preference, $F(1,4529)=4.22, p=.04$; RTs were longer when participants chose a synthetic form than an analytic form.

4.4. SUMMARY OF REACTION TIME RESULTS. When participants were unprimed, there were no significant differences in RTs for the synthetic versus the analytic comparative form for all three preference groups. Similarly, when participants were primed with a base-only prime, there were no significant differences in RTs for the synthetic versus the analytic form, or in comparison with the unprimed study, for all three preference groups. ${ }^{7}$

When primed with a same-synthetic prime, participants had significantly shorter RTs when they chose the analytic comparative form for -er-preference and no-preference adjectives, compared to (1) when primed with a same-synthetic prime and choosing a synthetic form, or (2) when unprimed and choosing either comparative form. For the more-preference adjectives, RTs were significantly shorter than when unprimed, but there were no significant differences in RTs for the synthetic versus the analytic form.

When primed with a different-synthetic prime, there were no significant differences in RTs for the synthetic versus the analytic form for all three preference groups. However, there was a significant difference in RTs between preference groups: A one-way ANOVA was conducted to examine the effect of preference group on RTs for -er-preference, no-preference, and morepreference adjectives that were primed with a different-synthetic prime. There was a statistically significant effect of preference group on RT, $F(2,2790)=17.12, p<.001$. Post-hoc comparisons using the Tukey HSD test indicated that the mean RTs for -er-preference $(\mathrm{M}=.262, \mathrm{~S}=0.938)$ and no-preference $(\mathrm{M}=.352, \mathrm{~S}=.921)$ adjectives were significantly higher than the mean $\mathrm{RT}$ for more-preference adjectives $(\mathrm{M}=.107, \mathrm{~S}=.786)$. Additionally, all three preference groups had significantly greater RTs than the same preference group in the unprimed study.

The reaction time results for the unprimed study and each of the three primed studiessame-synthetic, different-synthetic, and base only—are illustrated in Figure 9.

5. Why does recency only influence high-frequency adjectives? In support of previous research (Graziano King 1999, Adams 2014), Experiment 1 shows that frequency influences English comparative form preferences; specifically, high-frequency adjectives of some prosodic shapes (monosyllabic adjectives and disyllabic adjectives ending in $y$ ) are preferred in the synthetic comparative form.

According to Adams (2014), this occurs because frequency influences the prosodic representation of the adjective. This prosodic representation of high-frequency adjectives allows for -er suffixation at both the stem and word strata. Contrastingly, low-frequency adjectives only permit -er suffixation at the word stratum. As a result, high-frequency adjectives form the comparative synthetically more often than low-frequency adjectives. Adams writes, "I propose that this dual-membership behavior of comparative -er induces quantitative asymmetries in -er suffixation: the earlier -er can attach, the higher the rate of -er suffixation observed for a particular adjective" (2014: 166). In other words, an adjective's frequency influences its prosodic representations, which influences its rate of -er suffixation.

\footnotetext{
${ }^{7}$ Although there are no significant differences in RTs for the base-only primed study, the RTs for no-preference adjectives in the synthetic form seem to be longer than those in the analytic form. Similarly, the acceptabilityjudgment task results for this group differs (though not significantly) from the unprimed study (see Section 3.4). This seems to suggest that priming may be affecting this group in some way.
} 

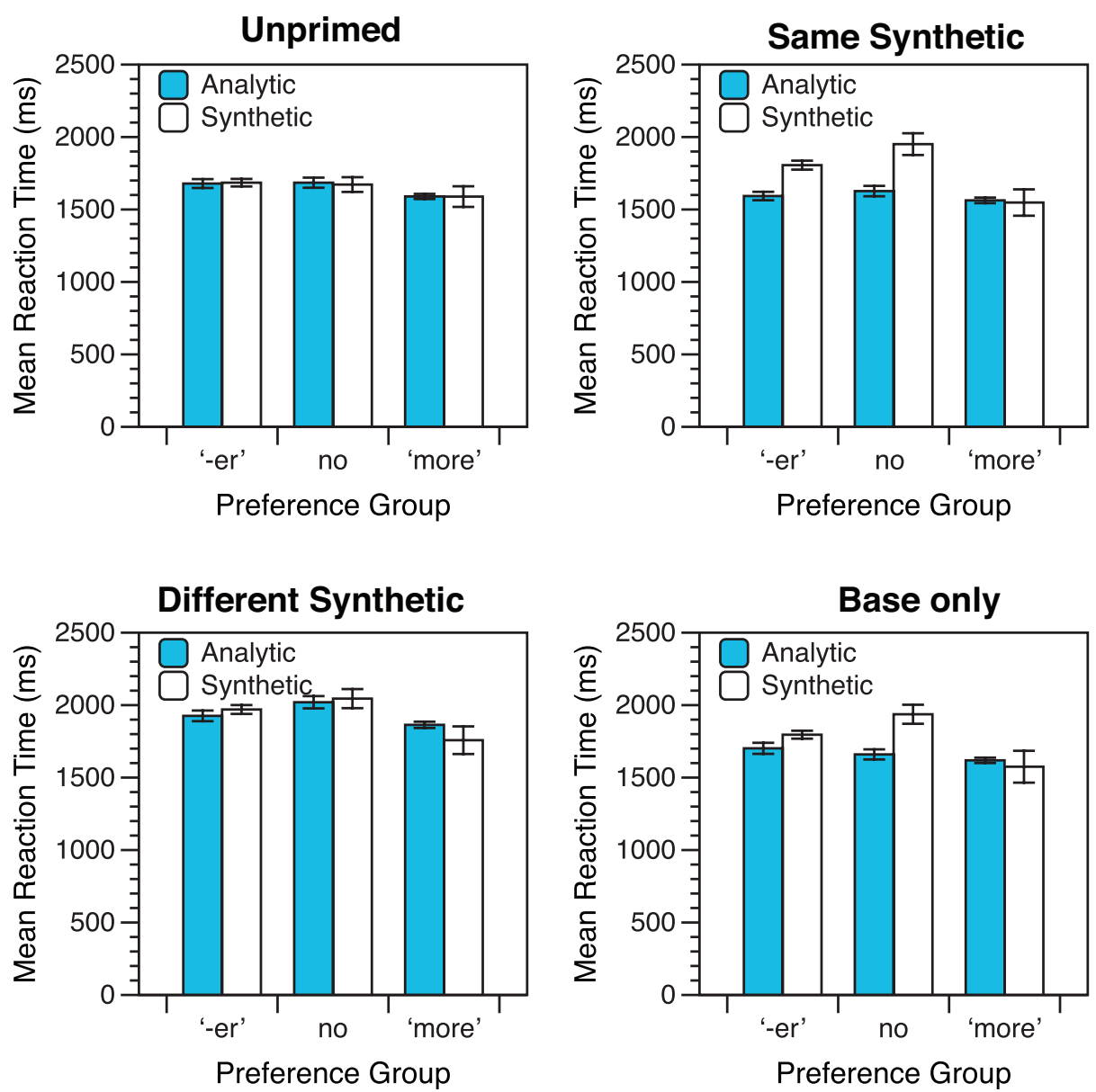

Figure 9. RTs (and standard error) by preference group and comparative preference for the unprimed study and the same-synthetic, different-synthetic, and base-only primed studies

In Experiment 2, recency had the greatest influence on high-frequency adjectives; lowfrequency adjectives were not affected. This result supports Adams's (2014) argument that the prosodic representation of an adjective permits more or less -er suffixation. More specifically, recency influenced high-frequency adjectives because more suffixation was permitted, while recency did not influence low-frequency adjectives because restrictions related to the adjective's prosodic shape blocked recency from impacting speaker preferences in the same manner that it did for high-frequency adjectives.

6. Conclusion. This research shows that, in addition to prosodic shape and frequency, recency of a synthetic comparative influences comparative form selection. However, synthetic form recency influences comparative form selection differently depending on an adjective's preference group. For -er-preference and no-preference adjectives, recency decreases selection of the synthetic form. For more-preference adjectives, recency increases selection of the synthetic form.

These results suggest that recency affects speakers' preferences differently depending on a speaker's experience with an adjective. If a speaker has experience with an adjective in the synthetic comparative form (-er-preference and no-preference adjectives), recency of a synthetic comparative causes inhibition of the synthetic comparative form. If a speaker does not have ex- 
perience with the adjective in the synthetic comparative form (more-preference adjectives), recency causes facilitation of the synthetic form.

This finding supports the idea that more-frequent forms (in this case, synthetic comparative forms for -er-preference and no-preference adjectives) are stored in a speaker's lexicon and accessed differently than less-frequent forms (in this case, synthetic comparative forms for morepreference adjectives). For -er-preference and no-preference adjectives, speakers have the synthetic form stored and, thus, are able to inhibit the synthetic form, causing some of the variation that occurs for these types of adjectives. For more-preference adjectives, however, speakers do not have enough experience with these adjectives in the synthetic form to store those forms; therefore, they are unable to inhibit the synthetic form. Instead, recency of these adjectives in the synthetic comparative form facilitates use of the adjective in the synthetic form, causing speakers to learn and possibly store the synthetic form.

\section{References}

Aronoff, Mark. 1976. Word formation in generative grammar. Cambridge: MIT Press.

Ballinger, Pamela. 1991. Curioser and curioser: Usage of comparative -er in American and British English. In California Lingwistik Notes, vol. 3. California State University Department of Linguistics.

Bauer, Laurie. 1994. Watching English change:An introduction to the study of linguistic change in standard Englishes in the twentieth century. London: Longman.

Cygan, Jan. 1975. Synthetical comparatives in English. Bulletin de la Societé Polonaise de Linguistique, 33. 53-57.

Davies, Mark. 2008-. The Corpus of Contemporary American English (COCA): 520 million words, 1990-present. http://corpus.byu.edu/coca/.

Graziano-King, Janine. 1999. Acquisition of comparative forms in English. New York: CUNY dissertation.

Jespersen, Otto. 1949. A Modern English Grammar. Copenhagen: Einar Munksgaard.

Leech, Geoffrey N. \& Jonathan Culpeper. 1997. The comparison of adjectives in recent British English. In Terttu Nevalainen \& Leena Kahlas-Tarkka (eds.), To Explain the Present: Studies in the Changing English Language in Honour of Matti Rissanen, 353-373. Helsinki: Société Néophilologique.

Lindquist, Hans. 2000. Livelier or more lively? Syntactic and contextual factors influencing the comparison of disyllabic adjectives. Language and Computers, 30. 125-132.

Mayr, Susanne, \& Axel Buchner. 2007. Negative priming as a memory phenomenon. Zeitschrift für Psychologie/Journal of Psychology, 215(1). 35-51. https://doi.org/10.1027/00443409.215.1.35

Pennington, Jeffrey, Richard Socher, \& Christopher D. Manning. 2014. Glove: Global vectors for word representation. Proceedings of the Empirical Methods in Natural Language Processing (EMNLP 2014). 1532-1543. http://dx.doi.org/10.3115/v1/D14-1162

Raveh, Michal. 2002. The contribution of frequency and semantic similarity to morphological processing. Brain and Language, 81(1). 312-325. http://dx.doi.org/10.1006/brln.2001.2527

Sandhaus, Evan. 2008. The New York Times annotated corpus. Linguistic Data Consortium. 